Anaesthesist 2022 $\cdot$ 71:190-192 https://doi.org/10.1007/s00101-022-01105-y Angenommen: 9. Februar 2022

Online publiziert: 25 . Februar 2022

๑) Der/die Autor(en) 2022

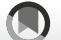

\title{
Zwanzigjähriges Jubiläum der Interdisziplinären Arbeitsgemeinschaft für Klinische Hämotherapie
}

\author{
Ein Grund zum Feiern!
}

\author{
Thomas Frietsch \\ Interdisziplinäre Arbeitsgemeinschaft für Klinische Hämotherapie, Marburg, Deutschland
}

Transfusionsbeauftragte, Transfusionsverantwortliche und Qualitätsbeauftragte Hämotherapie als wichtige klinische Funktionsträger sind in ihren Einrichtungen für die korrekte Ausführung der Hämotherapie, also der Therapie mit zellulärem Blut und aus Blutplasma hergestellten Blutprodukten, zuständig. Die besondere Rolle dieser Therapie wird deutlich, wenn man sich die jährliche Häufigkeit der therapeutischen Anwendungen verdeutlicht: Im Jahr 2020 wurden in Deutschland ca. 3,2 Mio. Erythrozytenkonzentrate, 480.000 Thrombozytenkonzentrate, 630.000 Plasmakonserven sowie eine Vielzahl aus Spenden gewonnene Gerinnungs- und Immunglobulinpräparate [5] zur vitalen Therapie von Patienten eingesetzt. Die fachgerechte Anwendung dieser wertvollen lebensrettenden Produkte erfordert spezielles Wissen und Erfahrung aus mehreren Disziplinen der Medizin.

Die Interdisziplinäre Arbeitsgemeinschaft für klinische Hämotherapie (IAKH) - als Interessenvertretung der Transfusionsverantwortlichen, Transfusionsbeauftragten und Qualitätsbeauftragten Hämotherapie - begleitet und unterstützt die Kliniker nun schon seit 20 Jahren.

\section{I) Von Beginn an widmet sich die IAKH der Fort-, Weiterbildung und Unterstützung klinisch tätiger Ärzte}

Am 16.02.2002 wurde auf den Marburger Transfusionsgesprächen mit der IAKH eine Arbeitsgemeinschaft gegründet [6], die durch die Verabschiedung des Trans- fusionsgesetzes (TFG) 1998 [2] und die Veröffentlichung der „Richtlinien zur Gewinnung von Blut und Blutbestandteilen und zur Anwendung von Blutprodukten (Hämotherapie)" [7] im Juli 2000 notwendig geworden war. Dort waren Qualifikation und Aufgaben von Transfusionsverantwortlichen, Transfusionsbeauftragten, Qualitätsbeauftragten Hämotherapie und der Transfusionskommission beschrieben und mussten erstmalig verpflichtend für die Einrichtungen bestellt werden. Ihre Tätigkeit und Qualifikation muss jährlich erfasst und zusammen mit dem restlichen Status der Qualität bei der Anwendung von Blutprodukten der zuständigen Landesärztekammer gemeldet werden.

In dieser Situation widmete sich die IAKH gemäß Satzung vordringlich der Fortbildung und Weiterbildung der klinisch tätigen Ärzte und auch der Unterstützung bei aktuellen Fragen und Problemen in der Klinik. Sie richtete mehrere Veranstaltungen pro Jahr aus, die als "Marburger Transfusionsgespräche" bekannt wurden. Treibende Kraft in den Anfangstagen war der in der klinischen Hämotherapie engagierte Internist, Transfusionsmediziner und Leiter des Instituts für Transfusionsmedizin und Hämostaseologie am Universitätsklinikum Marburg, Prof. Dr. med. Volker Kretschmer.Durch seine Beharrlichkeit und sein Engagement gelang es ihm damals schon im Schulterschluss mit der Landesärztekammer Hessen und vielen Anästhesisten eine hämotherapeutische Fachkompetenz bei vielen Klinikern herbeizuführen. Ein persönliches Hauptanliegen von Prof. 
Kretschmer war bereits vor der IAKH-Gründung 2002 die generelle Beratung aller Kliniker in transfusionsmedizinischen und hämostaseologischen Fragen.

An seiner Seite fanden sich von Anfang an Anästhesisten und Intensivmediziner. Sie haben Erfahrung mit der Anwendung von Blutkonserven - nicht nur bei herzchirurgischen oder sonstigen blutverbrauchenden Operationen. Durch die immense Patientennachfrage nach Eigenblut im Zuge des Skandals über Infektionen durch HIV-kontaminierte Blutprodukte der 1990er-Jahre hatten die Anästhesisten seit Jahren im Rahmen der Herstellung und des Betriebs von Eigenblutspendeeinrichtungen erheblichen transfusionsmedizinischen Sachverstand erworben. Unter allen Beteiligungen ist der fachdisziplinäre Beitrag der in der IAKH vertretenen Anästhesisten bis heute am größten. In der ersten Stunde waren mit Prof. Dr. med. Eberhard Götz aus Darmstadt, Dr. med. Arnulf Weiler-Lorentz aus Mannheim und Dr. med. Gerhard Wittenberg aus Ludwigshafen engagierte Anästhesisten als Mitstreiter im Boot. Das damalige Gründungs- mitglied Prof. Dr. med. Rainer Moosdorf, der ehemalige herzchirurgische Chef an der Uniklinik Marburg, ist heute noch im aktiven Vorstand der IAKH. Beliebte und geschätzte Referenten auf den Transfusionsgesprächen der IAKH waren Anästhesisten wie Prof. Dr. med. Jürgen Biscoping vom St. Vincentius Krankenhaus Karlsruhe, der kürzlich verstorbene Prof. Dr. med. Hans Gombotz aus dem Universitätsklinikum Graz, Prof. Dr. med. Donat Spahn aus Zürich und Prof. Dr. med. Sibylle Kietaipl aus Wien.

Die Hilfestellung in der klinischen Tätigkeit durch die IAKH war und ist für viele bestellte Ärzte bis heute notwendig und wird dankbar angenommen. Die Blutspendedienste betrachten ihre Aufgabe als pharmazeutischer Hersteller mit der Abgabe der Blutkonserven als beendet. Die IAKH hilft bis heute dort, wo kein anderer sich zuständig fühlt, und nimmt ihren Platz in der medizinischen Fachgesellschaft ein. Durch die große Erfahrung vieler Anästhesisten mit autologen Techniken, die heute kaum mehr eine Rolle spielen, wie normovolämischer Hämodilution, kontrollierter Hy- potension sowie Eigenblutspende unter Eisen- und Erythropoetineinsatz, wurden bereits sehr früh blutsparende Techniken im Sinne des heutigen „Patient Blood Management " betrieben und praktiziert. Die maschinelle Autotransfusion ist nach wie vor ein Hauptaugenmerk der IAKH, wie die jüngste Metaanalyse zum Einsatz bei onkologischen Eingriffen zeigt [4]. Die führende Rolle der Anästhesisten beim Aufbau von PBM-Ambulanzen wird v. a. in mittleren und kleineren Häusern durch praktische Anleitungen, Empfehlungen und Ratschläge durch die IAKH gestützt.

\section{》) Das satzungsgemäße Fördern der Qualität der Hämotherapie hat inzwischen viele Gesichter angenommen}

In den letzten 20 Jahren hat sich die IAKH substanziell weiterentwickelt. Das satzungsgemäße Fördern der Qualität der Hämotherapie hat viele Gesichter angenommen, zeitgemäß auf der Internetseite der IAKH (www.iakh.de) zugänglich. Dort werden den Mitgliedern Musterformu-

Hier steht eine Anzeige. 
lare und Fortbildungen zur Verfügung gestellt, Kontakte angezeigt und im Forum Fragen beantwortet, die die Arbeit als Transfusionsverantwortliche, Transfusionsbeauftragte oder Qualitätsbeauftragte Hämotherapie erheblich erleichtern. Die IAKH ist im Arbeitskreis Blut vertreten, hat sich bei der Anhörung von Neufassungen der Richtlinie und der QuerschnittsLeitlinien der Bundesärztekammer eingebracht. Sie hat als Verfahrenseigner des Qualitätssicherungsverfahrens „Peer Review Hämotherapie" den kollegialen Besuch zur Verbesserung der Hämotherapiepraxis erheblich geprägt und betreibt seit 2009 ein nationales kostenfreies Fehlerregister für die Anwendung von Blut- und Gerinnungsprodukten in Kooperation mit dem CIRSmedical der Bundesärztekammer. In unregelmäßigen Abständen bezieht die IAKH Stellung zu aktuellen Themen in der Hämotherapie wie z. B. der Vergütung und Freistellung der hämotherapeutischen Funktionsträger.

Neuere Initiativen sind die Ausschreibung und Verleihung eines Förderpreises, der Forschungsprojekte mit erheblicher Bedeutung für die klinische Hämotherapie unterstützt. Die Entwicklung einer software- und scannergestützten Absicherungstechnik bei der Anwendung von Blutkonserven und Plasmaderivaten durch die Arbeitsgruppe Transfusionssicherheit folgt der Zielsetzung eines der letzten Voten des Arbeitskreises Blut [1] zur Vermeidung von Fehltransfusionen und Verwechslungen. Eine computergestützte Simulationsschulung der Massivtransfusion in den anfordernden Kliniken hat pandemiebedingt noch zu wenig Auslastung erfahren. Für die meisten Ziele hat die IAKH Arbeitsgruppen gebildet. Weitere Initiativen umfassen die Mitarbeit im neuen Masterplan des Medizinstudiums, die Schließung einer faktischen Meldelücke für folgenlose Fehltransfusionen, die Entwicklung von Techniken zur Messbarkeit der Transfusionsindikation und vieles mehr.

\section{॥ Die IAKH wird auch weiterhin eine interdisziplinäre Plattform \\ für Kliniker, Labor- und Transfusionsmediziner anbieten}

Ganz entscheidend für die breite Akzeptanz der IAKH ist und bleibt die
Interdisziplinarität nicht nur zwischen Anästhesisten sowie Transfusions- und Labormedizinern, sondern sind und bleiben auch die Kontakte zu Hämatologen, Onkologen, Kardiologen, Chirurgen sowie anderen klinischen Hämotherapeuten und deren Fachgesellschaften. In den letzten Jahren bestand auch überwiegend eine gute Zusammenarbeit mit den Landesärztekammern in ganz Deutschland und der Bundesärztekammer sowie dem PaulEhrlich-Institut. Intensive Partnerschaften konnten mit dem Arbeitsausschluss Bluttransfusion der Deutschen Gesellschaft für Anästhesiologie und Intensivmedizin (DGAI)/dem Berufsverband Deutscher Anästhesisten (BDA), der Deutschen Interdisziplinären Vereinigung für Intensivund Notfallmedizin (DIVI), der Gesellschaft für Thrombose- und Hämostaseforschung (GTH), dem Network for the Advancement of Patient Blood Management, Haemostasis and Thrombosis (NATA) und dem Aktionsbündnis Patientensicherheit (APS) eingegangen werden. Trotz guter und enger Zusammenarbeit mit den industriellen Sponsoren mit besonderer Bedeutung für die Hämotherapie wahrt die IAKH ihre Neutralität und Objektivität in Fach- und Präparatefragen.

Abschließend kann sich die deutsche Medizin beglückwünschen, mit der IAKH eine Institution verfügbar zu haben, die immer noch ehrenamtlich geführt und trotz eines geringen Mitgliedsbeitrags ein hohes und attraktives Leistungsangebot für den engagierten Hämotherapeuten hat. Die breite Anerkennung der Leistung von Hämotherapeuten ist ein noch nicht erreichtes Ziel der Gesellschaft. Bluttransfusion und Gerinnungstherapie als lebenswichtige Therapien wollen gut durch Fachkompetenz abgesichert sein, um sicherzustellen, dass die speziellen Produkte in geeigneter Dosis am richtigen Patienten zum richtigen Zeitpunkt angewandt werden. Wie sehr dies notwendig ist, zeigen die Berichte und Empfehlungen aus dem Fehlerregister der IAKH [3]. Die IAKH schaut anlässlich ihres Jubiläums stolz auf die 20 Jahre ihres Bestehens zurück und fühlt sich verpflichtet, weiterhin eine Plattform für den Dialog und die Fortbildung von Klinikern, Labormedizinern und Transfusionsmedizinern anzubieten.

\section{Korrespondenzadresse}

Prof. Dr. Thomas Frietsch

Interdisziplinäre Arbeitsgemeinschaft für

Klinische Hämotherapie

An den Lahnbergen, 35043 Marburg,

Deutschland

frietsch@iakh.de

Interessenkonflikt. T. Frietsch gibt an, dass kein Interessenkonflikt besteht.

Open Access. Dieser Artikel wird unter der Creative Commons Namensnennung 4.0 International Lizenz veröffentlicht, welche die Nutzung, Vervielfältigung, Bearbeitung, Verbreitung und Wiedergabe in jeglichem Medium und Format erlaubt, sofern Sie den/die ursprünglichen Autor(en) und die Quelle ordnungsgemäß nennen, einen Link zur Creative Commons Lizenz beifügen und angeben, ob Änderungen vorgenommen wurden.

Die in diesem Artikel enthaltenen Bilder und sonstiges Drittmaterial unterliegen ebenfalls der genannten Creative Commons Lizenz, sofern sich aus der Abbildungslegende nichts anderes ergibt. Sofern das betreffende Material nicht unter der genannten Creative Commons Lizenz steht und die betreffende Handlung nicht nach gesetzlichen Vorschriften erlaubt ist, ist für die oben aufgeführten Weiterverwendungen des Materials die Einwilligung des jeweiligen Rechteinhabers einzuholen.

Weitere Details zur Lizenz entnehmen Sie bitte der Lizenzinformation auf http://creativecommons.org/ licenses/by/4.0/deed.de.

\section{Literatur}

1. Arbeitskreis Blut (2019) Stellungnahme Fehlanwendungen von Blutkomponenten. Bundesgesundheitsbl 62:1140-1143

2. Bundesministerium der Justiz sowie Bundesamts für Justiz. Gesetz zur Regelung des Transfusionswesens (Transfusionsgesetz). 1989, https://www. gesetze-im-internet.de/tfg/

3. Frietsch T, Thomas D, Schöler M et al (2017) Administration safety of blood products-lessons learned from a national registry for transfusion and hemotherapy practice. Transfus Med Hemother 44:240-254

4. Frietsch $T$, Steinbicker $A U$, Hackbusch $M$ et al (2020) Safety of cell salvage in tumor surgery: systematic review with meta-analysis. Anaesthesist 69:331-351

5. HenselerO (2021) Bericht desPaul-Ehrlich-Instituts über die nach $\S 21$ Transfusionsgesetz gemeldeten Daten 2020. http://www.pei.de/tfg-21 (p 21). Zugegriffen: 26.01.2022

6. Kretschmer V (2002) Interdisziplinäre Arbeitsgemeinschaft für KlinischeHämotherapiegegründet. Anaesth Intensivmed 43:382

7. Richtlinien zur Gewinnung von Blut und Blutbestandteilen und zur Anwendung von Blutprodukten (Hämotherapie). Bundesgesundheitsbl 2000;43(7):555-589 